\title{
Nurturing a sustainable Open Tree of Life
}

\author{
Emily Jane McTavish ${ }^{\ddagger}$, Mark Holder§, Karen Cranstonl \\ ‡ University of California, Merced, Merced, United States of America \\ $\S$ University of Kansas, Lawrence, United States of America \\ | Agriculture and Agri-food Canada, Ottawa, Canada
}

Corresponding author: Emily Jane McTavish (ejmctavish@ucmerced.edu)

Received: 11 Apr 2018 | Published: 21 May 2018

Citation: McTavish E, Holder M, Cranston K (2018) Nurturing a sustainable Open Tree of Life. Biodiversity

Information Science and Standards 2: e25727. https://doi.org/10.3897/biss.2.25727

\section{Abstract}

The Open Tree of Life project is a collaborative effort to synthesize, share and update a comprehensive tree of life Fig. 1. We have completed a draft synthesis of a tree summarizing digitally available taxonomic and phylogenetic knowledge for all 2.6 million named species, available at tree.opentreeoflife.org Hinchliff et al. 2015. . . This tree provides ready access to phylogenetic information which can link together biodiversity data on the basis of what we know about relevant evolutionary history. Both the unified reference taxonomy Rees and Cranston 2017 and the published phylogenetic statements underlying the tree McTavish et al. 2015 are available and accessible online. Taxa in the phylogenies are mapped to the the reference taxonomy, which aligns Open Tree taxon identifiers to those from NCBI and GBIF, among several other taxonomy resources. The synthesis tree is revised as new data become available, and captures conflict and consensus across different published phylogenetic estimates. This undertaking requires both development of novel infrastructure and analysis tools, as well as community engagement with the Open Tree of Life project. I will discuss the challenges in and the progress towards achieving these goals.

\section{Presenting author}

Emily Jane McTavish 


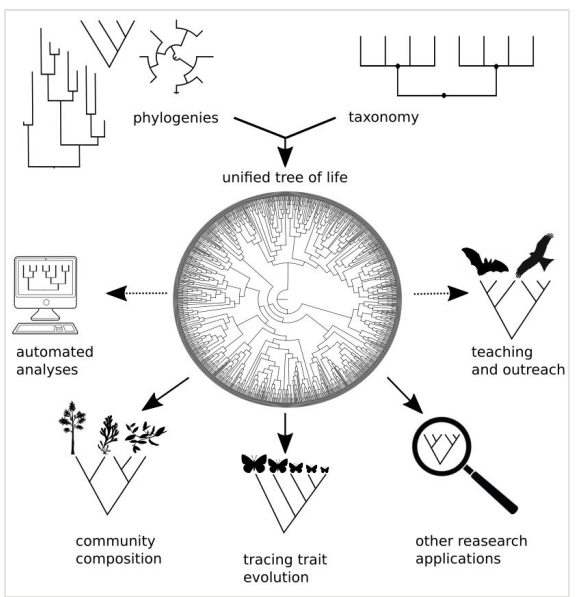

Figure 1.

A schematic of the process of building a unified tree of life. Inputs are published phylogenetic estimates and a unified taxonomy. These inputs are combined into a summary supertree containing over 2 million taxa. The relationships among any subset of taxa may easily be accessed and used in downstream analyses. Silhouettes from phylopic.org. Figure from McTavish et al. 2017

\section{Presented at}

TDWG 2018 - Biodiversity Information Standards Meeting

Dunedin, New Zealand

\section{References}

- Hinchliff C, Smith S, Allman J, Burleigh JG, Chaudhary R, Coghill L, Crandall K, Deng J, Drew B, Gazis R, Gude K, Hibbett D, Katz L, Laughinghouse HD, McTavish EJ, Midford P, Owen C, Ree R, Rees J, Soltis D, Williams T, Cranston K (2015) Synthesis of phylogeny and taxonomy into a comprehensive tree of life. Proceedings of the National Academy of Sciences 112 (41): 12764-12769. https://doi.org/10.1073/pnas.1423041112

- McTavish EJ, Hinchliff C, Allman J, Brown J, Cranston K, Holder M, Rees J, Smith S (2015) Phylesystem: a git-based data store for community-curated phylogenetic estimates. Bioinformatics 31 (17): 2794-2800. https://doi.org/10.1093/bioinformatics/ btv276

- McTavish EJ, Drew B, Redelings B, Cranston K (2017) How and Why to Build a Unified Tree of Life. BioEssays 39 (11): . https://doi.org/10.1002/bies.201700114

- Rees J, Cranston K (2017) Automated assembly of a reference taxonomy for phylogenetic data synthesis. Biodiversity Data Journal https://doi.org/10.3897/ BDJ.5.e12581 